\title{
AN EXTENSION OF HENRICI THEOREM FOR THE JOINT APPROXIMATE SPECTRUM OF COMMUTING SPECTRAL OPERATORS
}

\author{
ALI BEN AMOR \\ (Received 4 April 2001; revised 15 July 2002) \\ Communicated by A. Pryde
}

\begin{abstract}
Given two $m$-tuples of commuting spectral operators on a Hilbert space, $T=\left(T_{1}, \ldots, T_{m}\right)$ and $S=$ $\left(S_{1}, \ldots, S_{m}\right)$, an extended version of Henrici perturbation theorem is obtained for the joint approximate spectrum of $S$ under perturbation by $T$. We also derive an extended version of Bauer-Fike theorem for such tuples of operators. The method used involves Clifford algebra techniques introduced by McIntosh and Pryde.
\end{abstract}

2000 Mathematics subject classification: primary 47A13; secondary 34L05.

Keywords and phrases: spectral operator, joint spectrum, Henrici's bound.

\section{Introduction}

The study of tuples of commuting operators was the subject of a wide literature carrying out many resemblances with the single case. Various kinds of spectra were introduced, namely by Harte [10, 11], Taylor [21] and Coburin and Schechter [6]. Even more, Taylor [20] has introduced a functional calculus for tuples of operators, while Harte [11] established 'Spectral mappings theorems' for them. For more about joint spectral theory, the reader is referred to the survey paper of Curto [7].

Later on many authors focused on perturbations of tuples of operators. The use of analytic 'multi-functions' enabled Klimek [13] to prove the results known for single analytic perturbations. The turning point in this theory was the formulation by McIntosh and Pryde [14] of a functional calculus using tools from Clifford Analysis. Using these tools, Pryde [18] generalized the theorem of Bauer-Fike for commuting

(c) 2003 Australian Mathematical Society 1446-8107/03 \$A2.00+0.00 
tuples of matrices, while those of Henrici and Hoffman-Wielandt were extended by Bhatia and Bhattacharyya [3,2]. We also note that other properties were proved by Ming [16] and Müller [17].

Our aim in this paper is to extend Henrici [19, Theorem 1.9, page 172] and BauerFike [19, Theorem 1.6, page 171] theorems for tuples of commuting spectral operators defined on a complex Hilbert space. In fact, we establish bounds similar to the matrix case given by Pryde [18] and by Bhatia-Bhattacharyya [3] which are inspired by the Bauer-Fike and Henrici bounds for single matrices. This will be done using Clifford analysis.

The paper is organized as follows: Following [18], we first give some necessary tools and definitions, then we establish the Henrici theorem for tuples of operators having real spectra. At the end we consider operators with complex spectra.

\section{Preliminaries}

We denote by $H$ a nontrivial complex Hilbert space and by $\mathbb{B}(H)$ the algebra of bounded linear operators on $H$ with respect to the uniform norm. All the operators considered in this paper are in $\mathbb{B}(H)$. We will denote by $\mathbb{R}$, (respectively $\mathbb{C}$ ) the scalar field of real, (respectively of complex) numbers.

Let us consider an $m$-tuple of commuting operators: $T=\left(T_{1}, \ldots, T_{m}\right)$, where $T_{j} \in \mathbb{B}(H), T_{j} T_{k}=T_{k} T_{j}$ for $j, k=1,2, \ldots, m$. The joint approximate spectrum of $T$ is defined by:

$$
\operatorname{Sp}_{\text {ap }}(T)=\left\{\lambda=\left(\lambda_{1}, \ldots, \lambda_{m}\right) \in \mathbb{C}^{m} ; \inf _{\|x\|=1}\left\{\sum_{1 \leq j \leq m}\left\|T_{j} x-\lambda_{j} x\right\|\right\}=0\right\} .
$$

For a single operator $T$, the approximate spectrum will be denoted by $\sigma_{\text {ap }}(T)$. The joint compression spectrum (or right spectrum) of $T$ is defined by

$$
\operatorname{Sp}_{\mathrm{com}}(T)=\left\{\lambda=\left(\lambda_{1}, \ldots, \lambda_{m}\right) \in \mathbb{C}^{m}, \sum_{1 \leq j \leq m}\left(T_{j}-\lambda_{j} I\right) \mathbb{B}(H) \neq \mathbb{B}(H)\right\} .
$$

The set of joint eigenvalues of $T$ which we denote by $\operatorname{Sp}_{p}(T)$ is defined as follows: $\lambda=\left(\lambda_{1}, \ldots, \lambda_{m}\right) \in \operatorname{Sp}_{p}(T)$ if there is $x \in H, x \neq 0$ such that $T_{j} x=\lambda_{j} x$ for $j=1,2, \ldots, m$. One then has $\operatorname{Sp}_{p}(T) \subset \operatorname{Sp}_{\text {ap }}(T)$. For a single operator $T, \operatorname{Sp}_{p}(T)$ will be denoted by $\sigma_{p}(T)$.

Naturally the joint spectrum of $T$ is $\operatorname{Sp}(T)=\operatorname{Sp}_{\text {ap }}(T) \cup \operatorname{Sp}_{\text {com }}(T)$. We mention that the definitions of spectra are those of Harte [10], so the joint spectra considered in this paper are the Harte spectra. Let us emphasize that it is known $[4,7]$ that $\operatorname{Sp}_{\text {ap }}(T)$ is a compact nonempty subset of $\mathbb{C}^{m}$. 
We are always interested to know whether or not an operator has eigenvalues. In case of general commuting tuples of operators we do not know so far of any answer. However, if the operators are algebraic or compact having certain property, then the answer is affirmative. We recall that an operator $T \in \mathbb{B}(H)$ is algebraic if there is a nontrivial polynomial $P$ such that $P(T)=0$.

PROPOSITION 2.1. Let $T=\left(T_{1}, \ldots, T_{m}\right)$ be a commuting tuple of compact operators. Suppose that each $T_{k}$ has at least one nonzero point in its spectrum, then $\operatorname{Sp}_{p}(T) \neq \emptyset$.

PROOF. Pick $0 \neq \lambda_{1,1} \in \sigma_{p}\left(T_{1}\right)$, then there is $x \in H, x \neq 0$ with $T_{1} x-\lambda_{1,1} x=0$. Now, by assumption and by the properties of compact operators, there is $v_{2} \in \mathbb{N}^{*}, j_{2} \in$ $\mathbb{N}^{*}$ such that $\left(T_{2}-\lambda_{j_{2}, 2} I\right)^{\nu_{2}-1} x \neq 0$ and $\left(T_{2}-\lambda_{j_{2} ; 2}\right)^{\nu_{2}} x=0$.

Put $u_{1}=x, u_{2}=\left(T_{2}-\lambda_{j_{2}, 2} I\right)^{\nu_{2}-1} u_{1}$, then $T_{2} u_{2}-\lambda_{j_{2}, 2} u_{2}=0$ and $T_{1} u_{2}-\lambda_{1,1} u_{2}=0$. By induction we construct a joint eigenvector: suppose we have $u_{m-1}$, then $T_{k} u_{m-1}-$ $\lambda_{j_{k}, k} u_{m-1}=0$ for $k=1, \ldots, m-1$ with $j_{1}=1$. Then there is $v_{m}, j_{m}$ such that $\left(T_{m}-\lambda_{j_{m}, m} I\right)^{v_{m}-1} u_{m-1} \neq 0$ and $\left(T_{m}-\lambda_{j_{m}, m} I\right)^{v_{m}} u_{m-1}=0$. Set

$$
u_{m}=\left(T_{m}-\lambda_{j_{m}, m} I\right)^{v_{m}-1} u_{m-1},
$$

then

$$
T_{m} u_{m}-\lambda_{j_{m}, m} u_{m}=0=T_{m-1} u_{m}-\lambda_{j_{m-1}, m-1} u_{m}=\cdots=T_{1} u_{m}-\lambda_{1,1} u_{m} .
$$

Thereby $\left(\lambda_{1,1}, \ldots, \lambda_{j_{m}, m}\right) \in \operatorname{Sp}_{p}(T)$.

For algebraic operators the proof is essentially the same.

Since the tool we use is Clifford analysis we now make a brief incursion into it. Let $\left(e_{1}, \ldots, e_{m}\right)$ be the canonical basis of $\mathbb{R}^{m}$. The Clifford algebra $\mathbb{R}_{m}$ over $\mathbb{R}^{m}$ is the real algebra of maximal dimension with identity 1 containing $\mathbb{R}$ and $\mathbb{R}^{m}$ as subalgebras and such that $x^{2}=-\|x\|^{2}$ for every $x \in \mathbb{R}^{m}$. It follows that $e_{k}^{2}=-1$ and $e_{k} e_{j}=-e_{j} e_{k}$ for $k \neq j$. Now we set $e_{\emptyset}=1$ and if $E=\left\{s_{1}, \ldots, s_{k}\right\} \subset\{1, \ldots, m\}$, define $e_{E}=(-1)^{\sigma} e_{t_{1}} \cdots e_{t_{k}}=(-1)^{\sigma} e_{t_{1} \cdots t_{k}}$, where $1 \leq t_{1}<\cdots<t_{k}$ and $\sigma$ is the number of inversions in the permutation

$$
\pi=\left(\begin{array}{lll}
t_{1} & \cdots & t_{k} \\
s_{1} & \cdots & s_{k}
\end{array}\right)
$$

Clearly $\mathbb{R}_{m}$ has dimension $2^{m}$ and has a basis consisting of $e_{E}, E=\emptyset$ or $E=$ $\left\{s_{1}, \ldots, s_{k}\right\}$ with the $s_{j}$ 's ordered in a natural way.

A full description of the real Clifford algebras $\mathbb{R}_{m}$ for $m \leq 4$ is given in [8]. There $\mathbb{R}_{m}$ is seen under many aspects, involution and conjugation are constructed over it. We recall that $\mathbb{R}_{1}$ is isomorphic to both $\mathbb{C}$, the matrix algebra $S O(2)$ and to $\operatorname{Spin}(2)$, the 
spinor real algebra; $\mathbb{R}_{2}$ is isomorphic to $\bar{H}$ the quaternions algebra and to the matrix algebra $S U(2)$. For $m=3, \mathbb{R}_{3}$ is isomorphic to $\bar{H} \oplus \bar{H}$ and $\mathbb{R}_{4}$ is isomorphic to $\bar{H}(2)$, the algebra of $2 \times 2$ matrices over $\bar{H}$. For more details the reader is referred to [8].

We define a new Hilbert space denoted by $H \otimes \mathbb{R}_{m}$ whose elements are of the form $\sum_{S} x_{S} \otimes e_{S}, x_{S} \in H, S \subset\{1, \ldots, m\}$ endowed with the inner product: $(x, y)=$ $\sum_{S, S^{\prime}}\left(x_{S}, y_{S^{\prime}}\right)$ for $x, y \in H \otimes \mathbb{R}_{m}$ and the norm $\|x\|^{2}=\sum_{S}\left\|x_{S}\right\|^{2}$. Let $\mathbb{B}\left(H \otimes \mathbb{R}_{m}\right)$ denote the algebra of bounded linear operators on $H \otimes \mathbb{R}_{m}$ together with the operator norm $\|\cdot\|$. Then $\mathbb{B}(H) \otimes \mathbb{R}_{m}$ may be identified with a sub-algebra of $\mathbb{B}\left(H \otimes \mathbb{R}_{m}\right)$ as follows:

$$
\left(\sum_{S} T_{S} \otimes e_{S}\right)\left(\sum_{S^{\prime}} x_{S^{\prime}} \otimes e_{S^{\prime}}\right)=\sum_{S, S^{\prime}} T_{S}\left(x_{S^{\prime}}\right),
$$

so that $\mathbb{B}(H) \subset \mathbb{B}(H) \otimes \mathbb{R}_{m} \subset \mathbb{B}\left(H \otimes \mathbb{R}_{m}\right)$. For a commuting $m$-tuple of $\mathbb{B}(H)$, $T=\left(T_{1}, \ldots, T_{m}\right)$ we define the Clifford operator of $T$ by

$$
\operatorname{Cliff}(T)=i \sum_{1 \leq j \leq m} T_{j} \otimes e_{j} \in \mathbb{B}(H) \otimes \mathbb{R}_{m} \subset \mathbb{B}\left(H \otimes \mathbb{R}_{m}\right) .
$$

If $x \in H$, then

$$
\text { Cliff(T) } x=i \sum_{1 \leq j \leq m} T_{j} x \otimes e_{j} .
$$

We also define the adjoint of Cliff $(T)$ by

$$
\operatorname{Cliff}(T)^{*}=i \sum_{1 \leq j \leq m} T_{j}^{*} \otimes e_{j} .
$$

Later on we denote by $\|\operatorname{Cliff}(T)\|$ the operator norm of $\operatorname{Cliff}(T)$ and by $\gamma(T)$ the spectral set

$$
\gamma(T)=\left\{\lambda=\left(\lambda_{1}, \ldots, \lambda_{m}\right) \in \mathbb{R}^{m}, \sum_{1 \leq j \leq m}\left(T_{j}-\lambda_{j} I\right)^{2} \text { is singular }\right\} .
$$

McIntosh and Pryde proved in [14] that $\gamma(T)=\operatorname{Sp}(T) \cap \mathbb{R}^{m}$, and if the $T_{j}$ 's have real spectra, then $\gamma(T)=\operatorname{Sp}(T)$. In this situation $0 \notin \operatorname{Sp}(T)$ if and only if $\operatorname{Cliff}(T)$ is regular and

$$
(\operatorname{Cliff}(T))^{-1}=\left(\sum_{1 \leq j \leq m} T_{j}^{2}\right)^{-1} \operatorname{Cliff}(T) .
$$

We also define the joint spectral radius of $T$ :

$$
r(T)=\max \{|\lambda|, \lambda \in \operatorname{Sp}(T)\} .
$$


PROPOSITION 2.2. Let $T=\left(T_{1}, \ldots, T_{m}\right)$ be an m-tuple of commuting bounded operators, where every $T_{j}$ is normal, then

(a) $\operatorname{Cliff}(T)$ is normal.

(b) $r(\operatorname{Cliff}(T))=r(T)$.

(c) $\|\operatorname{Cliff}(T)\|=r(T)$.

The proof is the same as the one of [18, Proposition 3.2], so we omit it.

We are now in a position to give the desired generalization of the results given by Pryde [18] and Bhatia and Bhattacharyya [3]. Following them we first consider perturbations of real spectra then of complex spectra.

\section{Perturbations of real spectra}

The class of operators in which we are interested are spectral operators:

DEFINITION 3.1 ([9, page 1939]). We say that a bounded operator $T$ is a spectral operator if it is the sum $T=S+N$ of a scalar operator $S$ and a quasi-nilpotent operator $N$ commuting with $S$.

For the definition of a scalar and quasi-nilpotent operator the reader is referred to [9]. It is known that such decomposition of a spectral operator is unique and is called the canonical decomposition of $T$. For a single spectral operator $T=S+N$, it is known that $\sigma(T)=\sigma(S)$. We will first extend this property for tuples of spectral operators having real spectra.

PROPOSITION 3.2. Let $T=\left(T_{1}, \ldots, T_{m}\right)$ be a commuting tuple of spectral operators having real spectra. For $1 \leq j \leq m$, let $\Lambda_{j}$ be the scalar part of the canonical decomposition of $T_{j}$ and $N_{j}$ its quasi-nilpotent part. Set $\Lambda=\left(\Lambda_{1}, \ldots, \Lambda_{m}\right)$, then $\operatorname{Sp}(T)=\operatorname{Sp}(\Lambda)$.

ProOF. It is known [14, Proposition 10.1] that $\operatorname{Sp}(T)=\gamma(T)$, so $\lambda=\left(\lambda_{1}, \ldots\right.$, $\left.\lambda_{m}\right) \in \operatorname{Sp}(T)$ if and only if $0 \in \sigma\left(\sum_{1 \leq j \leq m}\left(T_{j}-\lambda_{j} I\right)^{2}\right)$. The latter operator is equal to

$$
\sum_{1 \leq j \leq m}\left(\Lambda_{j}-\lambda_{j} I\right)^{2}-2 \sum_{1 \leq j \leq m}\left(\Lambda_{j}-\lambda_{j} I\right) N_{j}+\sum_{1 \leq j \leq m} N_{j}^{2}
$$

Since the operator $2 \sum_{1 \leq j \leq m}\left(\Lambda_{j}-\lambda_{j} I\right) N_{j}+\sum_{1 \leq j \leq m} N_{j}^{2}$, is quasi-nilpotent and commutes with the operator $\sum_{j=1}^{m}\left(\Lambda_{j}-\lambda_{j} I\right)^{2}$, then $0 \in \sigma\left(\sum_{1 \leq j \leq m}\left(T_{j}-\lambda_{j} I\right)^{2}\right)$ if and only if $0 \in \sigma\left(\sum_{1 \leq j \leq m}\left(\Lambda_{j}-\lambda_{j} I\right)^{2}\right)$ which means that $\lambda \in \gamma(S)=\operatorname{Sp}(S)$ and this completes the proof. 
From now on, the symbols $\Lambda$ and $S$ have the meaning specified in Proposition 3.2.

The first relevant result we are going to establish is an extension of Bauer-Fike theorem [19, page 171].

Proposition 3.3. Let $T=\left(T_{1}, \ldots, T_{m}\right)$ and $S=\left(S_{1}, \ldots, S_{m}\right)$ be two $m$-tuples of commuting operators. If $\lambda \in \mathrm{Sp}_{\mathrm{ap}}(S) \backslash \mathrm{Sp}(T)$ and $A \in \mathbb{B}(H)$ is regular then

$$
\left\|A^{-1}[\operatorname{Cliff}(T-\lambda I)]^{-1} A\right\|^{-1} \leq\left\|A^{-1} \operatorname{Cliff}(T-S) A\right\| .
$$

Proof. By the hypothesis, there is a sequence $\left(x_{n}\right) \subset H,\left\|x_{n}\right\|=1$ such that

$$
\sum_{j=1}^{m}\left\|\left(S_{j}-\lambda_{j} I\right) x_{n}\right\| \rightarrow 0
$$

and $\operatorname{Cliff}(T-\lambda I)$ is regular. Now

$$
\begin{aligned}
\operatorname{Cliff}(T-\lambda I) x_{n} & =i \sum_{j=1}^{m}\left(T_{j}-\lambda_{j} I\right) x_{n} \otimes e_{j} \\
& =\operatorname{Cliff}(T-S) x_{n}+\operatorname{Cliff}(S-\lambda I) x_{n},
\end{aligned}
$$

which leads to

$$
x_{n}=[\operatorname{Cliff}(T-\lambda I)]^{-1} \operatorname{Cliff}(T-S) x_{n}+[\operatorname{Cliff}(T-\lambda I)]^{-1} \operatorname{Cliff}(S-\lambda I) x_{n} .
$$

Inserting $A A^{-1}=I$, we get

$$
\begin{aligned}
x_{n}= & A A^{-1}[\operatorname{Cliff}(T-\lambda I)]^{-1} A A^{-1} \operatorname{Cliff}(T-S) A A^{-1} x_{n} \\
& +A A^{-1}[\operatorname{Cliff}(T-\lambda I)]^{-1} \operatorname{Cliff}(S-\lambda I) x_{n}
\end{aligned}
$$

and then

$$
\begin{aligned}
\left\|A^{-1} x_{n}\right\| \leq & \left\|A^{-1}[\operatorname{Cliff}(T-\lambda I)]^{-1} A\right\|\left\|A^{-1} \operatorname{Cliff}(T-S) A\right\|\left\|A^{-1} x_{n}\right\| \\
& +\left\|A^{-1}[\operatorname{Cliff}(T-\lambda I)]^{-1} \operatorname{Cliff}(S-\lambda I) x_{n}\right\| .
\end{aligned}
$$

From (3.1) follows that

$$
\begin{aligned}
\limsup _{n \rightarrow \infty}\left\|A^{-1} x_{n}\right\| \leq & \left\|A^{-1}[\operatorname{Cliff}(T-\lambda I)]^{-1} A\right\| \\
& \times\left\|A^{-1} \operatorname{Cliff}(T-S) A\right\| \limsup _{n \rightarrow \infty}\left\|A^{-1} x_{n}\right\| .
\end{aligned}
$$

Since $\left\|A^{-1} x_{n}\right\| \leq\left\|A^{-1}\right\|$ and lim $\sup _{n \rightarrow \infty}\left\|A^{-1} x_{n}\right\|>0$ we get

$$
1 \leq\left\|A^{-1}[\operatorname{Cliff}(T-\lambda I)]^{-1} A\right\|\left\|A^{-1} \operatorname{Cliff}(T-S) A\right\|,
$$

which completes the proof. 
In the remaining part of this section we suppose that all the operators under consideration have real spectra.

Now we are going to give the version of Henrici's bound extended to our situation, generalizing thereby the result stated by Bhatia and Bhattacharyya [3, Theorem 3.2] to the case of tuples of spectral operators. This will be done without defining the measure of nonnormality as it was the case in [3].

THEOREM 3.4. Let $T=\left(T_{1}, \ldots, T_{m}\right)$ and $S=\left(S_{1}, \ldots, S_{m}\right)$ be two $m$-tuples of commuting spectral operators. Let $T_{j}=\Lambda_{j}+N_{j}$ be the canonical decomposition of the $T_{j}$ 's, where $\Lambda_{j}$ is scalar and $N_{j}$ is quasi-nilpotent. Then given $\beta \in \operatorname{Sp}_{\mathrm{ap}}(S) \backslash \mathrm{Sp}(T)$, there is a self-adjoint bounded invertible operator B such that for

$$
K=\left[B \operatorname{Cliff}(\Lambda-\beta I) B^{-1}\right]^{-1} B \operatorname{Cliff}(N) B^{-1},
$$

we have

$$
\frac{\delta}{\left\|(I+K)^{-1}\right\|} \leq\left\|B \operatorname{Cliff}(T-S) B^{-1}\right\|
$$

where $\delta=\min \{|\alpha-\beta|, \alpha \in \operatorname{Sp}(T)\}$.

ProOF. Let $\beta \in \mathrm{Sp}_{\text {ap }}(S) \backslash \mathrm{Sp}(T)$, then by Proposition 3.2, $\beta \in \mathrm{Sp}_{\text {ap }}(S) \backslash \mathrm{Sp}(\Lambda)$. Now the $\Lambda_{j}$ 's is a family of commuting scalar operators, hence by [9, Theorem 4 , page 1947], there is a bounded invertible self-adjoint operator $B$ such hat $B \Lambda_{j} B^{-1}$ are normal, hence $B\left(\Lambda_{j}-\beta_{j} I\right) B^{-1}$ are also normal. A direct computation gives:

$$
\begin{aligned}
& B \operatorname{Cliff}(T-\beta I) B^{-1} \\
& \quad=B \operatorname{Cliff}(\Lambda-\beta I) B^{-1}\left[I+\left[B \operatorname{Cliff}(\Lambda-\beta I) B^{-1}\right]^{-1} B \operatorname{Cliff}(N) B^{-1}\right] .
\end{aligned}
$$

Put $K=\left[B \operatorname{Cliff}(\Lambda-\beta I) B^{-1}\right]^{-1} B \operatorname{Cliff}(N) B^{-1}$, then $K$ is quasi-nilpotent. Thus $I+K$ is invertible and we have

$$
B[\operatorname{Cliff}(T-\beta I)]^{-1} B^{-1}=(I+K)^{-1} B[\operatorname{Cliff}(\Lambda-\beta I)]^{-1} B^{-1} .
$$

Now put $\mu=\left\|B[\operatorname{Cliff}(\Lambda-\beta I)]^{-1} B^{-1}\right\|^{-1}$, then we get

$$
\frac{\mu}{\left\|(I+K)^{-1}\right\|} \leq\left\|B[\operatorname{Cliff}(T-\beta I)]^{-1} B^{-1}\right\|^{-1} .
$$

Hence using Proposition 3.3, we get

$$
\frac{\mu}{\left\|(I+K)^{-1}\right\|} \leq\left\|B \operatorname{Cliff}(T-S) B^{-1}\right\| .
$$

It remains to prove that $\mu=\delta=\min \{|\alpha-\beta|, \alpha \in \operatorname{Sp}(T)\}$. 
Since $B\left(\Lambda_{j}-\beta_{j} I\right) B^{-1}=\tilde{\Lambda}_{j}$ are normal for $j=1, \ldots, m$ then by Proposition 2.2, $B \operatorname{Cliff}(\Lambda-\beta I) B^{-1}$ is normal, and thereby $B[\operatorname{Cliff}(\Lambda-\beta I)]^{-1} B^{-1}$ is also normal. It follows that

$$
\left\|B[\operatorname{Cliff}(\Lambda-\beta I)]^{-1} B^{-1}\right\|=r\left(B[\operatorname{Cliff}(\Lambda-\beta I)]^{-1} B^{-1}\right) .
$$

Since $B[\operatorname{Cliff}(\Lambda-\beta I)]^{-1} B^{-1}$ and $[\operatorname{Cliff}(\Lambda-\beta I)]^{-1}$ have the same joint spectra, we get $\mu=\max \left\{|\xi|^{-1}, \xi \in \sigma(\operatorname{Cliff}(\Lambda-\beta I))\right\}$. Observing that $\sigma(\operatorname{Cliff}(\Lambda-\beta I))=$ $\gamma(\Lambda-\beta I)$, we get

$$
\mu=\max \left\{|\xi|^{-1}, \xi \in \gamma(\Lambda-\beta I)\right\}=\max \left\{|\lambda-\beta|^{-1}, \xi \in \gamma(\Lambda)=\operatorname{Sp}(\Lambda)\right\} .
$$

Recall that by Proposition 3.2, we have $\operatorname{Sp}(\Lambda)=\operatorname{Sp}(T)$, which implies that $\mu=\max \left\{|\lambda-\beta|^{-1}, \lambda \in \gamma(\Lambda)=\operatorname{Sp}(T)\right\}=\delta$, which completes the proof.

In case where the operators are self-adjoint we have $\operatorname{Sp}_{\text {ap }}(T)=\mathrm{Sp}(T)$. Indeed, if $\lambda=\left(\lambda_{1}, \ldots, \lambda_{m}\right) \notin \mathrm{Sp}_{\mathrm{ap}}(T)$ then there is $S_{1}, \ldots, S_{m}$ in $\mathbb{B}(H)$ such that $\left(T_{1}-\right.$ $\left.\lambda_{1} I\right) S_{1}+\cdots+\left(T_{m}-\lambda_{m} I\right) S_{m}=I$, hence $S_{1}^{*}\left(T_{1}-\lambda_{1} I\right)+\cdots+S_{m}^{*}\left(T_{m}-\lambda_{m} I\right)=I$, thus $\lambda \notin \mathrm{Sp}(T)$. On the other hand, every self-adjoint operator is a spectral operator whose quasi-nilpotent part is zero. Thus, in Theorem 3.4 we have $K=0$ and we can chose $B=I$. Applying Theorem 3.4 to both $T$ and $S$ (by symmetry) where $T$ and $S$ are tuples of commuting self-adjoint operators, we get

$$
\min \{|\alpha-\beta|, \alpha \in \operatorname{Sp}(T)\} \leq\|\operatorname{Cliff}(T-S)\|
$$

for every $\beta \in \operatorname{Sp}(S)$ and

$$
\min \{|\alpha-\beta|, \alpha \in \operatorname{Sp}(S)\} \leq\|\operatorname{Cliff}(T-S)\|
$$

for every $\beta \in \operatorname{Sp}(T)$. We have thus proved the following:

PROPOSITION 3.5. Let $T$ and $S$ be two $m$-tuples of commuting self-adjoint operators and let $\Delta(\operatorname{Sp}(T), \operatorname{Sp}(S))$ be the Hausdorff distance between $\operatorname{Sp}(T)$ and $\operatorname{Sp}(S)$. Then:

$$
\Delta(\operatorname{Sp}(T), \operatorname{Sp}(S)) \leq\|\operatorname{Cliff}(T-S)\| .
$$

EXAMPLE 1. Let us give an example were the bound (3.2) is attained. Let $P$ be a nontrivial self-adjoint projection. Take $T_{1}=P, T_{2}=I-P, T=\left(T_{1}, T_{2}\right)$ and $S=(0,0)$. Then $\operatorname{Sp}(T) \subset\{(0,0),(0,1),(1,0),(1,1)\}$. Since $T_{1}^{2}+T_{2}^{2}=$ $\left(T_{1}-I\right)^{2}+\left(T_{2}-I\right)^{2}=I$, we conclude that the points $(0,0)$ and $(1,1)$ do not belong to $\operatorname{Sp}(T)$. On the other hand, we have

$$
T_{1}^{2}+\left(T_{2}-I\right)^{2}=2 P, \quad \text { and } \quad\left(T_{1}-I\right)^{2}+T_{2}^{2}=2(I-P),
$$

which yields $\operatorname{Sp}(T)=\{(0,1),(1,0)\}$. Whence, $\Delta(\operatorname{Sp}(T), \operatorname{Sp}(S))=1=\|\operatorname{Cliff}(T)\|$. 
To visualize how Theorem 3.4 extends Henrici's theorem, known for tuples of matrices [3], we will consider a special case of operators. From the point of view of spectral properties the algebraic operators may be the 'nearest' operators to matrices.

Proposition 3.6. Let $T=\left(T_{1}, \ldots, T_{m}\right)$ and $S=\left(S_{1}, \ldots, S_{m}\right)$ be two $m$-tuples of commuting (respectively) algebraic and spectral operators. Let $T_{j}=\Lambda_{j}+N_{j}$ be the canonical decomposition of the $T_{j}$ 's where $\Lambda_{j}$ is scalar and $N_{j}$ is nilpotent. Then given $\beta \in \operatorname{Sp}_{\mathrm{ap}}(S) \backslash \mathrm{Sp}(T)$, there is a self-adjoint bounded invertible operator $B$ and an integer $v \in \mathbb{N}$ such that

$$
\frac{\delta}{\sum_{0 \leq k \leq \nu-1}\left(\left\|B \operatorname{Cliff}(N) B^{-1}\right\|^{k} / \delta^{k}\right)} \leq\left\|B \operatorname{Cliff}(T-S) B^{-1}\right\|,
$$

where $\delta=\min \{|\alpha-\beta|, \alpha \in \operatorname{Sp}(T)\}$.

REMARK. According to the proof of Theorem 3.4, if $T$ and $S$ are tuples of commuting matrices then $B$ can be chosen unitary and we thus get Henrici theorem for tuples of matrices [3, Theorem 3.2], with respect to the operator norm. If moreover $T$ is a single normal matrix then we get the Bauer-Fike theorem [1].

ProOF. Let $\beta \in \mathrm{Sp}_{\text {ap }}(S) \backslash \mathrm{Sp}(T)$, denote by $K$ the operator

$$
K=\left[B \operatorname{Cliff}(\Lambda-\beta I) B^{-1}\right]^{-1} B \operatorname{Cliff}(N) B^{-1} .
$$

Then $K=\sum_{k, p=1}^{m} \tilde{T}_{k} \tilde{N}_{p} \otimes e_{k} e_{p}$, where

$$
\tilde{T}_{k}=\left(\sum_{j=1}^{m}\left(B \Lambda_{j} B^{-1}-\beta_{j} I\right)^{2}\right)^{-1}\left(B \Lambda_{k} B^{-1}-\beta_{k} I\right)
$$

and $\tilde{N}_{p}=B N_{p} B^{-1}$. Hence $\tilde{N}_{p}$ is nilpotent and $\tilde{T}_{k} \tilde{N}_{p}=\tilde{T}_{k} \tilde{N}_{p}$ for $k, p=1, \ldots, m$ (this is because $\Lambda_{k} \tilde{N}_{p}=\tilde{N}_{p} \Lambda_{k}$ ). It follows that $\tilde{T}_{k} \tilde{N}_{p} \otimes e_{k} e_{p}$ is nilpotent for $k=1,2, \ldots, m$ and therefore $K$ is nilpotent: there is $v \in \mathbb{N}$ such that $K^{v}=0$. Thus $(I+K)^{-1}=\sum_{k=0}^{v-1}(-1)^{k} K^{k}$, which gives as in the last proof

$$
B[\operatorname{Cliff}(T-\beta I)]^{-1} B^{-1}=(I+K)^{-1} B[\operatorname{Cliff}(\Lambda-\beta I)]^{-1} B^{-1} ;
$$

this leads to the following inequality

$$
\begin{aligned}
\left\|B[\operatorname{Cliff}(T-\beta I)]^{-1} B^{-1}\right\| \leq & \sum_{k=0}^{\nu-1}\left\|B[\operatorname{Cliff}(\Lambda-\beta I)]^{-1} B^{-1}\right\|^{k} \\
& \times\left\|B \operatorname{Cliff}(N) B^{-1}\right\|^{k}\left\|B[\operatorname{Cliff}(\Lambda-\beta I)]^{-1} B^{-1}\right\| \\
= & \delta^{-1} \sum_{k=0}^{\nu-1} \frac{\left\|B \operatorname{Cliff}(N) B^{-1}\right\|^{k}}{\delta^{k}} .
\end{aligned}
$$


Then using Proposition 3.3 we get

$$
\delta\left(\sum_{k=0}^{v-1} \frac{\left\|B \operatorname{Cliff}(N) B^{-1}\right\|^{k}}{\delta^{k}}\right)^{-1} \leq\left\|B \operatorname{Cliff}(T-S) B^{-1}\right\| .
$$

\section{Perturbations of tuples with complex spectra}

Following Pryde [18], to remove the assumption of real spectra, we shall use partitions of tuples of operators. We shall also need the 'spectral mapping theorem' for both the joint spectrum and the joint approximate spectrum. We first recall the definition of a partition [18].

Definition 4.1. We say that an $m$-tuple $T=\left(T_{1}, \ldots, T_{m}\right)$ has an admissible partition if each $T_{j}$ can be written $T_{j}=T_{1 j}+i T_{2 j}$ where $T_{1 j}, T_{2 j}$ have real spectra and $\pi(T)=\left(T_{11}, \ldots, T_{1 m}, T_{21}, \ldots, T_{2 m}\right)$ is a $2 m$-tuple of commuting operators; $\pi(T)$ is called an admissible partition of $T$.

It was proved by Pryde and McIntosh [14, Theorem 10.8], that if $T=\left(T_{1}, \ldots, T_{m}\right)$ is an $m$-tuple of commuting operators having an admissible partition and if

$$
P: \mathbb{C}^{2 m} \rightarrow \mathbb{C}^{m}, P\left(\lambda_{1}, \ldots, \lambda_{2 m}\right)=\left(\lambda_{1}+i \lambda_{m+1}, \ldots, \lambda_{m}+i \lambda_{2 m}\right),
$$

then

$$
\operatorname{Sp}(T)=\operatorname{Sp}(P(\pi(T)))=P(\operatorname{Sp}(\pi(T)))=P(\gamma(\pi(T))) .
$$

This means that if $\lambda \in \operatorname{Sp}(T)$, then $(\operatorname{Re}(\lambda), \operatorname{Im}(\lambda)) \in \operatorname{Sp}(\pi(T)$, where $\operatorname{Re}(\beta), \operatorname{Im}(\beta)$ are respectively the real and the imaginary parts of $\beta$.

It is also known [15] that every tuple of commuting spectral operators has an admissible partition. Thus using this results one can carry over Proposition 3.2 to arbitrary tuples consisting of commuting spectral operators.

Taking this into account, in order to pass from the 'real case' to the 'complex case', we just need a 'spectral mapping theorem' for the joint approximate spectrum. Namely (see [5, Theorem 1, page 319] or [7, Corollary 3.7, page 43]):

Let $P: \mathbb{C}^{m} \rightarrow \mathbb{C}^{n}$ be a polynomial and $T$ an $m$-tuple of commuting operators, then

$$
P\left(\operatorname{Sp}_{\mathrm{ap}}(T)\right)=\mathrm{Sp}_{\mathrm{ap}}(P(T)) .
$$

It follows in particular, that if $P$ is the polynomial defined by (4.1) and if $\lambda \in \operatorname{Sp}_{\text {ap }}(T)$, then $(\operatorname{Re}(\lambda), \operatorname{Im}(\lambda)) \in \operatorname{Sp}_{\text {ap }}(\pi(T))$.

We are now able to establish some perturbation theorems for tuples having complex spectra. We adopt the following notation: If $\beta=\left(\beta_{1}, \ldots, \beta_{m}\right) \in \mathbb{C}^{m}$ we put

$$
\widetilde{\beta}=(\operatorname{Re}(\beta), \operatorname{Im}(\beta))=\left(\operatorname{Re}\left(\beta_{1}\right), \ldots, \operatorname{Re}\left(\beta_{m}\right), \operatorname{Im}\left(\beta_{1}\right), \ldots, \operatorname{Im}\left(\beta_{m}\right)\right) \in \mathbb{C}^{2 m} .
$$


THEOREM 4.2. Let $T=\left(T_{1}, \ldots, T_{m}\right)$ and $S=\left(S_{1}, \ldots, S_{m}\right)$ be two $m$-tuples of commuting spectral operators. Let $\pi(T)$ be an admissible partition of $T$, say $\pi(T)=$ $\left(T_{11}, \ldots, T_{1 m}, T_{21}, \ldots, T_{2 m}\right)$ and let $T_{k j}=\Lambda_{k j}+N_{k j}, k, j=1,2, \ldots, m$, be the canonical decomposition of the $T_{k j}$. Set $\pi(S)$ an admissible partition of $S, \pi(\Lambda)=$ $\left(\Lambda_{11}, \ldots, \Lambda_{1 m}, \Lambda_{21}, \ldots, \Lambda_{2 m}\right)$ and $\pi(N)=\left(N_{11}, \ldots, N_{1 m}, N_{21}, \ldots, N_{2 m}\right)$. Given $\beta \in \mathrm{Sp}_{\mathrm{ap}}(S) \backslash \mathrm{Sp}(T)$, there is a self-adjoint bounded invertible operator $B$ such that for $K=\left[B \operatorname{Cliff}(\pi(\Lambda)-\tilde{\beta} I) B^{-1}\right]^{-1} B \operatorname{Cliff}(\pi(N)) B^{-1}$, we have

$$
\frac{\delta}{\left\|(I+K)^{-1}\right\|} \leq\left\|B \operatorname{Cliff}(\pi(T)-\pi(S)) B^{-1}\right\|,
$$

where $\delta=\min \{|\alpha-\beta|, \alpha \in \operatorname{Sp}(T)\}$.

ProOF. By (4.2) and (4.3), one get that if $\beta \in \operatorname{Sp}_{\text {ap }}(S) \backslash \operatorname{Sp}(T)$ then $\widetilde{\beta} \in \operatorname{Sp}_{\text {ap }}(\pi(S)) \backslash$ $\operatorname{Sp}(\pi(T))$. Now applying Theorem 3.4, we get the result.

Using Theorem 4.2, we are going to carry over [18, Corollary 6.4] for tuples of commuting normal operators.

Proposition 4.3. Let $T=\left(T_{1}, \ldots, T_{m}\right)$ and $S=\left(S_{1}, \ldots, S_{m}\right)$ be two $m$-tuples of commuting normal operators whose compression spectra are invariant under the map $z \mapsto \bar{z}$. Let $\pi(T)$, respectively $\pi(S)$, be an admissible partition of $T$, respectively of $S$, and $\pi\left(S^{*}\right)$ the $2 m$-tuple of operators composed by the adjoints of the operators corresponding to $\pi(S)$. Denote by $\Delta(\mathrm{Sp}(T), \mathrm{Sp}(S))$ the Hausdorff distance between $\operatorname{Sp}(T)$ and $\operatorname{Sp}(S)$. Then

$$
\Delta(\operatorname{Sp}(T), \operatorname{Sp}(S)) \leq \max \left(\|\operatorname{Cliff}(\pi(S)-\pi(T))\|,\left\|\operatorname{Cliff}\left(\pi\left(S^{*}\right)-\pi(T)\right)\right\|\right) .
$$

Proof. Applying Theorem 4.2 to $S^{*}=\left(S_{1}^{*}, \ldots, S_{m}^{*}\right)$ with the partition $\pi\left(S^{*}\right)$, we get

$$
\max _{\beta \in \mathrm{SP}_{\mathrm{ap}}\left(S^{*}\right)} \min _{\alpha \in \mathrm{Sp}(T)}|\alpha-\beta| \leq\left\|\operatorname{Cliff}\left(\pi\left(S^{*}\right)-\pi(T)\right)\right\| .
$$

Now the approximate spectrum of $S^{*}$ is nothing else but the complex conjugate of the compression spectrum of $S$; this leads to

$$
\max _{\beta \in \operatorname{Sp}(S)} \min _{\alpha \in \operatorname{Sp}(T)}|\alpha-\beta| \leq \max \left(\|\operatorname{Cliff}(\pi(S)-\pi(T))\|,\left\|\operatorname{Cliff}\left(\pi\left(S^{*}\right)-\pi(T)\right)\right\|\right) .
$$

Interchanging $S$ and $T$, we get

(4.4) $\Delta(\operatorname{Sp}(T), \operatorname{Sp}(S)) \leq \max \left(\|\operatorname{Cliff}(\pi(S)-\pi(T))\|,\left\|\operatorname{Cliff}\left(\pi\left(S^{*}\right)-\pi(T)\right)\right\|\right.$,

$$
\left.\left\|\operatorname{Cliff}\left(\pi\left(T^{*}\right)-\pi(S)\right)\right\|\right) \text {. }
$$


Now by the definition of the adjoint of the Clifford operator we have

$$
\operatorname{Cliff}\left(\pi\left(S^{*}\right)-\pi(T)\right)=\left(\operatorname{Cliff}\left(\pi(S)-\pi\left(T^{*}\right)\right)\right)^{*} .
$$

Thereby the right-hand term in (4.4) is equal to

$$
\max \left(\|\operatorname{Cliff}(\pi(S)-\pi(T))\|,\left\|\operatorname{Cliff}\left(\pi\left(S^{*}\right)-\pi(T)\right)\right\|\right),
$$

which finishes the proof.

By similar arguments to those used in the proof of Theorem 4.2, one can prove the following result which is an extension of [18, Theorem 6].

PROPOSITION 4.4. Under the assumptions of Theorem 4.2, if

$$
\epsilon=\|\operatorname{Cliff}(\pi(T)-\pi(S))\|\|B\|\left\|B^{-1}\right\|,
$$

then $\operatorname{Sp}_{\text {ap }}(T) \subset \bigcup_{\beta \in \operatorname{Sp}(S)} D_{\epsilon}(\beta)$, where $D_{\epsilon}(\beta)$ is the disc centered at $\beta$ with radius $\epsilon$.

\section{Acknowledgment}

I am thankful to the referee for bringing the reference [7] into my attention.

\section{References}

[1] F. L. Bauer and C. T. Fike, 'Norms and exclusion theorems', Numer. Math. 2 (1960), 137-141.

[2] R. Bhatia and R. Bhattacharyya, 'A generalization of the Hoffman-Wielandt theorem', Linear Algebra Appl. 179 (1993), 11-17.

[3] — ' 'A Henrici theorem for joint spectra of commuting matrices', Proc. Amer. Math. Soc. 118 (1993), 5-14.

[4] J. Bunce, 'The joint spectrum of commuting nonnormal operators', Proc. Amer. Math. Soc. 29 (1977), 499-505.

[5] M. D. Choi and C. Davis, 'The spectral mapping theorem for joint approximate spectrum', Bull. Amer. Math. Soc. 20 (1974), 317-321.

[6] L. A. Coburin and M. Schechter, 'Joint spectra and interpolation of operators', J. Funct. Anal. 2 (1968), 226-237.

[7] R. E. Curto, 'Applications of several complex variables to multiparameter spectral theory', in: Surveys of some recent results in operator theory, Vol. II, Pitman Res. Notes Math. Ser. 192 (Longman Sci. Tech., Harlow, 1988) pp. 25-90.

[8] R. Delanghe, F. Sommen and V. Souček, Clifford algebra and spinor-valued functions. A function theory for the Dirac operator, Mathematics and its Applications 53 (Kluwer, Dordrecht, 1992).

[9] N. Dunford and J. T. Schwartz, Linear operators. Part III (Wiley, New York, 1971).

[10] R. Harte, 'The spectral mapping theorem in several variables', Bull. Amer. Math. Soc. 78 (1972), 871-875. 
[11] - 'Spectral mapping theorems', Proc. Roy. Irish Acad. Sec. A 72 (1972), 89-107.

[12] P. Henrici, 'Bounds for iterates, inverses, spectral variation and fields of values of non-normal matrices', Numer. Math. 4 (1962), 24-40.

[13] M. Klimek, 'Joint spectra and analytic set-valued functions', Trans. Amer. Math. Soc. 294 (1986), 187-196.

[14] A. McIntosh and A. J. Pryde, 'A functional calculus for several commuting operators', Indiana Univ. Math. J. 36 (1987), 21-439.

[15] A. McIntosh, A. J. Pryde and W. Ricker, 'Comparison of joint spectra for certain classes of commuting operators', Studia Math. 88 (1988), 23-36.

[16] F. Ming, 'Garske's inequality for an n-tuple of operators', Integral Equations Operator Theory 14 (1991), 787-793.

[17] V. Müller, 'Local behavior of operators', Functional Analysis and Operator Theory (Warsaw 1992), Banach Center Publ. 30 (Polish Acad. Sci., Warsaw, 1994) pp. 251-258.

[18] A. J. Pryde, 'A Bauer-Fike theorem for the joint spectrum of commuting matrices', Linear Algebra Appl. 173 (1992), 221-230.

[19] G. W. Stewart and J. Sun, Matrix perturbation theory (Academic Press, New York, 1990).

[20] J. L. Taylor, 'The analytic-functional calculus for several commuting operators', Acta Math. 125 (1970), 1-38.

[21] _ 'A joint spectrum for several commuting operators', J. Funct. Anal. 6 (1970), 172-191.

\section{Universität Bielefeld}

\section{Fakultät für Mathematik}

\section{Bielefeld}

\section{Germany}

e-mail: benamor@mathematik.uni-bielefeld.de 